Original Research Paper

\title{
An Efficient Combined Call Admission Control and Scheduling Algorithm to Improve Quality of Service for Multimedia Services Over Next Generation Wireless Networks
}

\author{
${ }^{1}$ G. Nantha Kumar and ${ }^{2}$ A. Arokiasamy \\ ${ }^{I}$ Department of CSE, Anjalai Ammal Mahalingam Engineering College, Kovilvenni, Tamil Nadu, India \\ ${ }^{2}$ Department of CSE, E.G.S. Pillay Engineering College, Nagapattinam, Tamil Nadu, India
}

Article history

Received: 14-05-2014

Revised: 22-08-2014

Accepted: 18-09-2014

Corresponding Author:

G. Nantha Kumar

Department of CSE, Anjalai Ammal

Mahalingam Engineering College,

Kovilvenni, Tamil Nadu, India

Email: gan_nand@yahoo.com

\begin{abstract}
We nominate a weighted service queuing approach, each service queue is allotted a preset priority weight and Call Admission Control scheme for service classes defined in IEEE 802.16 standard. Different group of queues have been implemented to transmit different service classes. Allocation of bandwidth usage of each service classes are relying on maximum data that could take part in connection between different networks. The Quality of Service is achieved by considering throughput and delay as a major parameters along with the corresponding service classes taken into account. The scheduled CAC model with the token bucket mechanism allows increasing the performance of Quality of Service for multimedia services. By applying the weighted queuing approach, the acceptance of new connection request is increased to achieve the better Quality of Service in multimedia services over next generation wireless networks.
\end{abstract}

Keywords: Service Class, Scheduled CAC Model, QoS, Weighted Service Queuing Approach

\section{Introduction}

Call admission control in multimedia wireless communications is to guarantee Quality of Service (QoS) to mobile users and to achieve high resource utilization efficiency. QoS parameters are guaranteed with $100 \%$ confidence. Typically, these schemes require extensive knowledge of the system parameters such as user mobility to satisfy the Quality of Service (QoS).

In the CAC algorithm new call arrival rates are estimated continuously and if they are higher than a predetermined level some calls are blocked irrespective of whether a channel is available or not. The objective of this scheme is to maintain the new call arrival rate lesser than a predetermined level.

The main function of call admission control algorithm is to limit the interference by controlling the number of new call accepted in the network. The role of call admission control algorithm is to ensure that admittance of a new flow into a resource constrained network does not violate service commitments made by the network to admitted flows. The service commitments made could be quantitative or it could be more qualitative.

CAC algorithm must determine the priority of this type of call over new calls. Use of CAC algorithm indicates that this algorithm reduces the drop of handoff calls and increases the total system capacity and performance; hence the grade of service of the system can significantly be improved especially in case of high mobility environments.

The fundamental idea behind all collaborative admission control schemes is to consider not only local information but also information in the network. The local cell, where the new call has been requested, communicates with a set of cells that will participate in the admission process. This set of cells is usually referred to a cluster (Ramesh Babu et al., 2010). 
Call admission control is very important measure in CDMA system to guarantee the quality of communicating links (Kolate et al., 2012). The call dropping happens in the network in most of the case when, accepted call that has not completed in the current cell may have to be handed off to another cell. During this process, the call may not be able to gain a channel in the new cell to continue its service due to the limited resource wireless networks (Kolate et al., 2012).

Thus, the new pulse and handoff calls have to be treated differently with priority in terms of resource allocation. Since users tend to be much more sensitive to call dropping than to be call blocking, handoff calls are normally assigned higher priority over the new calls (Deshmukh and Deshmukh, 2013). Handoff prioritization can improve handoff related system performances. Two handoff prioritization schemes guard channels and queuing (Kolate et al., 2012).

The Robust Call Admission Control (RCAC) was proposed in (Kumar and Arokiasamy, 2014) by concentrating on transmissions and space to provide the priority for the new request connections. This has been compared with previous model of CAC which could provide less performance measures for the future generation wireless heterogeneous networks. Based on the class of service taken from (Kumar and Arokiasamy, 2014), it was concentrated on the both real and non real time service categories.

IEEE 802.16 has supported the mesh distributed mode in (Rashidi et al., 2010) to introduce the connection between Subscriber Station with the Base Station using the average bit rate to achieve the Highest Performance of CAC algorithm implemented on the both stations. The guaranteed QoS was provided by the choice of choosing the bandwidth available between the stations. The acceptance and rejection of call request connection has been implemented in this (Rashidi et al., 2010) with Greedy algorithm.

Using bit rate, arrival rate and allotted bandwidths made the traffic intensity of the cellular networks in (Pla et al., 2008) to achieve the performance level of the users handling it. Some of the suboptimal policies have been considered to bring the performance of networks to the peel level as in (Pla et al., 2008).

New calls and handover calls normally have to be treated differently in terms of resource allocation. Hand over calls are normally assigned higher priority than new calls (Tragos et al., 2008).

In this study we intend to produce the weighted service queuing scheduling approach along with the Call Admission Control scheme for offering QoS over next generation wireless networks. The performance metrics has been considered to evaluate the functioning level of the scheme in wireless networks. The maximum volume of data to be sent can be calculated using token bucket mechanism and the naïve estimation of bandwidth is also under consideration. With the help of allocated bandwidth the packets can be transmitted by the local scheduler corresponds to the different queues related with different service classes. The performance is evaluated from the simulation results correspondingly.

The paper is organized as follows. Section II discuss about the system model that has been used in networks. WSQ scheduling algorithm and Call Admission Control with token bucket mechanism has been implemented in section III and IV respectively. Section V illustrates the evaluated simulation results with respect to performance measures and the paper concludes in section VI.

\section{System Model}

We consider the wireless networks standards can be configured in either single hop or muti-hop architecture where there are two specific components such as Base Station (BS) which offer internet access to the Subscriber Station (SS). In while, a connection has been established between end users with the help of services that are provided and requested. Resources are allocated on the basis of bandwidth estimation for each and every channel accessing relies on scheduling schemes. Conceiving the characteristics of traffic and requirements of QoS services, the wireless standard 802.16 classifies the services of connections into four different QoS groups: Unsolicited Grant Service (UGS), real time Polling Service (rtPS), non real time Polling Service (nrtPS) and Best Effort (BE).

The QoS architecture for the wireless standard network is shown in (Fathi et al., 2013) Fig. 1 and its corresponding service classes with the rated priority along with traffic type and application is shown in Table 1.

We consider uplink transmission in IEEE 802.16 standard where packets of a set of queues (Fathi et al., 2013) $Q=\{Q i: I=1,2, \ldots \ldots N\}$ are transmitted from a $\mathrm{SS}$ to BS. Four groups of queues are considered to transmit $Q_{U G S}, Q_{r t P S}, Q_{n r t P S}, Q_{B E}$ on behalf of four QoS classes defined in 802.16: UGS, rtPS, nrtPS, BE traffic.

About 802.16 use MAC option to be under preference condition and uses Time Division Duplex (TDD) or Frequency Division Duplex (FDD) to access the medium resource. 802.16 support QoS and divide all traffic flows into 4 classes based on their application type and different priority. 
Token Bucket is a mechanism which has been implemented in Call Admission Control (CAC) which having the capability for controlling the network traffic rate that has been injected to network which works well for the "bursty traffic" Fig. 2 shows how the mechanism acts upon.

The major two parameters have been followed in token bucket mechanism, bucket size ' $b$ ' and token rate ' $r$ '.

Each token represents a unit of bytes or a packet data unit. A packet is not allowed to be transmitted until it possesses a token. Therefore, over a period of time' $t$ ', the maximum data volume to be sent (Tsai and Jiang, 2006) will be:

$$
r * t+b
$$

We acquire the token bucket mechanism to schedule the packets in wireless standard network environments.

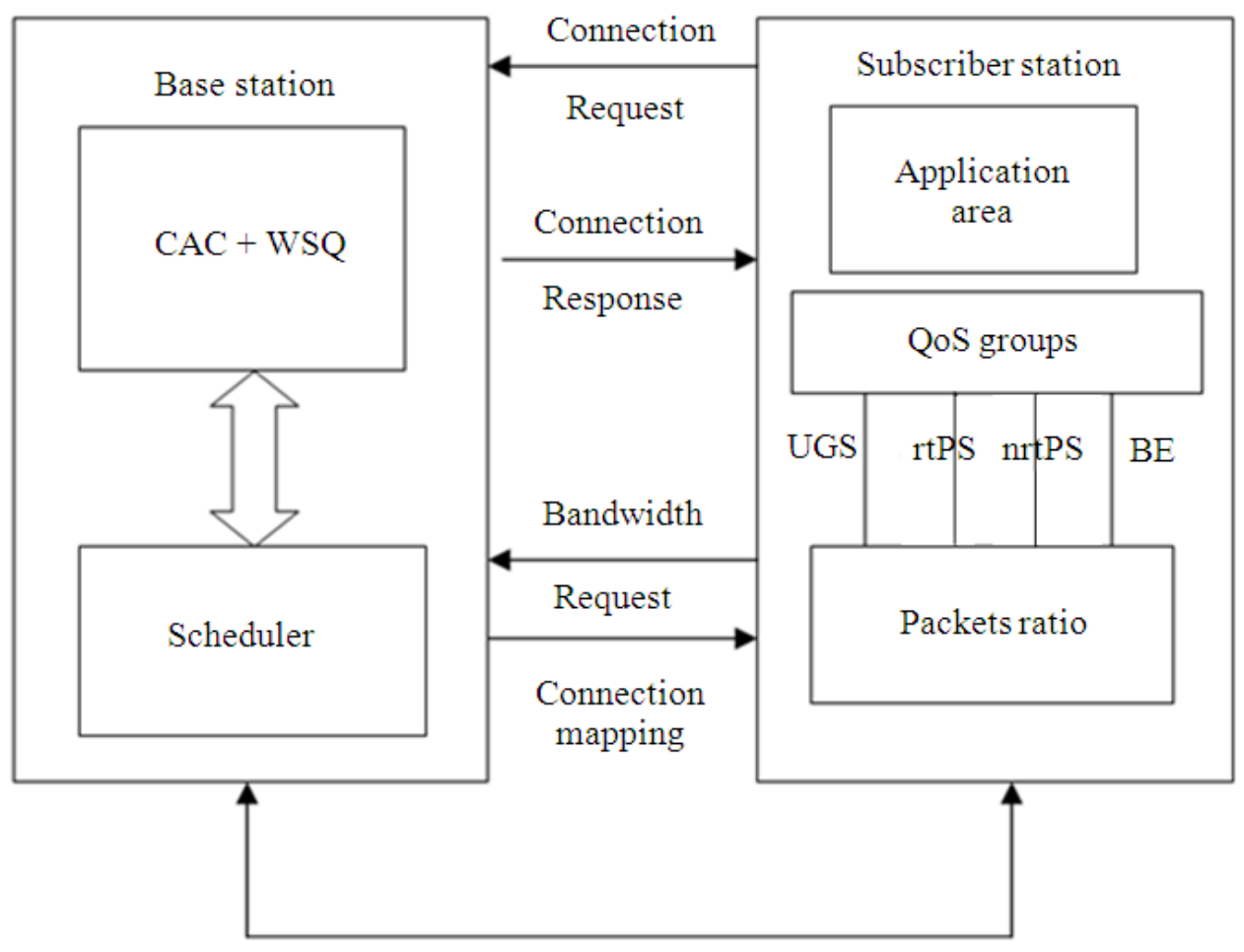

Fig. 1. System model for QoS groups

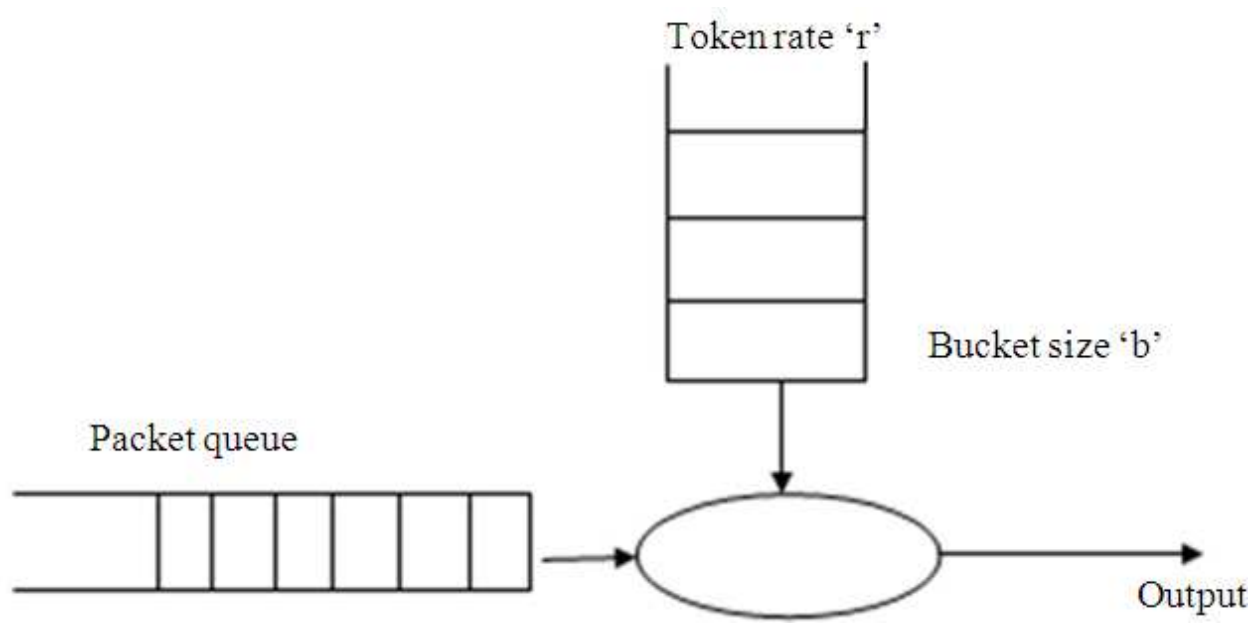

Fig. 2. Token bucket mechanism 


\begin{tabular}{lll}
\multicolumn{2}{l}{ Table 1. QoS classes } \\
\hline Class name & Traffic type & Application \\
\hline UGS & Real-time CBR & Voice over IP \\
rtPS & Real-time CBR & Real- time video \\
nrtPS & Non-real time traffic & FTP \\
Best effort & Non-real time traffic & HTTP
\end{tabular}

\section{Scheduling Scheme}

The proposed scheduling algorithm is to be discussed in this segment. Since UGS class having precedence under IEEE 802.16 standard, a predetermined amount of bandwidth is ever allocated to $\mathrm{C}_{\mathrm{UGS}}$ class. We assume the simulation time up to 60 seconds that has to be handled between Subscriber Station and Base Station. Therefore, we deal the bandwidth value from the following channel capacity Equation at time $\operatorname{slot}^{\prime} \mathrm{t}$ ' is given by Equation 1:

$$
\mathrm{C}^{\prime}(\mathrm{t})=\log _{2}(1+\gamma(\mathrm{t}))-\mathrm{C}_{\mathrm{UGS}} \mathrm{bps} / \mathrm{Hz}
$$

where, $\chi(t)$ is received signal to noise ratio is further used to allocate the bandwidth for the remaining service class rtPS, nrtPS and BE at every time slot ' $t$ '. This has been implemented by adopting the twoprocess scheduling scheme.

\section{Process I}

A predetermined priority weights i.e., $W_{r t P S}, W_{n r t P S}$, $\mathrm{W}_{\mathrm{BE}}$ allocated for each service queue on the basis of weighted service queuing approach as it stands in the order as Equation 2:

$$
\mathrm{W}_{\mathrm{rtPS}}>\mathrm{W}_{\mathrm{ntPS}}>\mathrm{W}_{\mathrm{BE}}
$$

Bandwidth allocation for each service class ' $s$ ' having rtPS, nrtPS, BE can be determined on the basis of priority function as follows:

$$
P_{s}(t)=\frac{W_{s}\left(1_{s}(t)+1\right) \lambda_{s}(t)}{r_{s}(t)} \text { for all 's' }
$$

$\mathrm{t} \quad=$ Time slot

$W_{s}=$ Priority weight

$l s(t)=$ Packet dropping probability

$\lambda_{s}(t)=$ Average arrival rates of class ' $s$ ' up to time slot ' $t$ '

$r_{s}(t)=$ Average departure rates of class ' $s$ ' up to time slot ' $t$ '

With obtained priority values from Equation 3, the bandwidth is to be allocated to each service class with help of following Equation as:
$B_{s}(t)=\frac{P_{s}(t) C^{\prime}(t)}{\sum_{i} P_{i}(t)}$ for all 's'

where, $i$ is given by list of services rtPS, nrtPS and BE respectively.

\section{WSQ Scheduling Scheme: Procedure I}

Compute priority values in Equation 3.

Compute $B_{s}(t)$; Bandwidth $S \in\{r t P S, n r t P S, \mathrm{~B} E\}$ as in Equation 4:

$$
\begin{aligned}
& \text { Compute } \mathrm{D}=\mathrm{r} * \mathrm{t}+\mathrm{b} ; \mathrm{t}->\text { token rate; } \mathrm{b}->\text { bucket size } \\
& \text { With DRR }=\mathrm{MP}_{\mathrm{S}}-\mathrm{P}_{\mathrm{L}} \text {; maximum packet size-Packet } \\
& \text { Length } \\
& \text { Handles packets for } \gamma_{r t P S}(t) \\
& \text { With FQ = at time' } \mathrm{t} \text { ' } \mathrm{R} / \mathrm{N} \text {-average data rate } \\
& \mathrm{R}->\text { data rate } \mathrm{N}->\text { data flow for } \gamma_{n r t P S}(t) \\
& \text { With SFQ = if } \text { Threshold }_{\text {Source }} \text { matches threshold } \\
& \text { G(n) = Random value for queuing packets for } \\
& \gamma_{B E}(t) \text { update each queue }
\end{aligned}
$$

\section{Process II}

Local schedulers have to be used to transmit packets in individual service classes one by one on the basis of obtained priority values in Process I. With the help of the Equation 4, each service classes are allocated with the respective bandwidth values. For each service classes rtPS, nrtPS and BE, with the help of Token Bucket mechanism, the maximum data volume to be sent using $\mathrm{r} * \mathrm{t}+\mathrm{b}$ is determined and taken along to the scheduling scheme following with the variety of queuing techniques that has been followed in the network simulator. We uses Deficit weighted Round Robin (DRR) scheduling, Fair Queuing (FQ) and Stochastic Fair Queuing (SFQ) for service classes $\gamma_{r t P S}(t), \quad \gamma_{n r t P S}(t), \quad \gamma_{B E}(t)$ respectively, which were supported by network simulator NS2. DRR schedules the varying size packets without having any idea about the mean size. It holds the packets which are taken from the concepts of maximum packet size number is subtracted from the packet length until it reaches the proper destination. Then, FQ uses to grant the various packet flows to fairly share the capacity of the available link. The average data rate with link data rate $\mathrm{R}$ for a given instant width $\mathrm{N}$ data flows can be serviced in this scheduling algorithm. Furthermore, SFQ scheme matches the source and destination threshold values based on the hash function mapping generated randomly for queuing the packets. WSQ scheme has been proposed under procedure I. 


\section{Scheduled CAC Scheme: Procedure II}

Upon arrival of a new connection $\mathrm{NC}$ at time slot ' $t$ ':

$$
\begin{aligned}
& \operatorname{Set}_{N C}(t)=0 \\
& \text { For } t=10 \text { to } T \rightarrow 60 \text { Seconds }
\end{aligned}
$$

Predict the values of $\gamma_{r t P S}(t), \gamma_{n r t P S}(t)$ and $\gamma_{B E}$ from the WSQ Scheduling Scheme:

$$
\begin{aligned}
& \Delta \gamma_{r t P S}(t)=\gamma_{r t P S}(t)-\gamma_{r t P S}^{T H} \\
& \Delta \gamma_{r t P S}(t)=\gamma_{r t P S}(t)-\gamma_{n r t P S}^{T H} \\
& \Delta \gamma_{B E}(t)=\gamma_{B E}(t)-\gamma_{B E}^{T H} \\
& \gamma_{N C}(t)=\gamma_{N C}(t)+1 / T\left(\Delta \gamma_{r t P S}(t)\right. \\
& \left.+\Delta \gamma_{n r t P S}(t)+\Delta \gamma_{B E}(t)\right)
\end{aligned}
$$

Then:

$$
\gamma_{\mathrm{NC}}(\mathrm{t})+10
$$

End for

If:

$$
\gamma_{N C}(t) \geq \gamma_{N C}^{T H}
$$

\section{Otherwise \\ Block NC \\ End}

Accept NC

\section{Scheduled CAC Scheme}

This scheme has been concentrated on the arrival of a new connection for given slot of time' $t$ ', here this slots of time taken up to 60 seconds. Based on the CAC scheme mentioned in (Fathi et al., 2013), we propose a scheduled CAC scheme by blending the queuing algorithms supported by the NS2 simulator which have been used for code implementation to measure the performances committed by the service classes to support the Quality of Services.

Initially the departure rate that is allocated to the new connection NC for a time slot' $t$ ' have been set to zero. Since the $\gamma_{r t P S}(t), \gamma_{n r t P S}(t)$ and $\gamma_{B E}(t)$ are the data rate of packets that has assigned to the service classes rtPS, nrtPS and BE respectively, can be predicted from the WSQ scheduling scheme applied in Procedure I. Each service classes $\gamma_{r t P S}, \gamma_{n r t P S}$ and $\gamma_{B E}$ has been assigned with the predetermined departure rate $\gamma_{r t P S}^{T H}, \gamma_{n r t P S}^{T H}$ and $\gamma_{B E}^{T H}$ respectively along with the threshold rate assigned for the arrival of new connection request.

From these predicted values, we compute the average value of data rate of packets that can be allocated to the service classes for a period of time' $t$ ' slots up to ' $T$ ' of seconds and also the data rate of packets for the arrival of new connection request $\gamma_{N C}(T)$ from using the Equation 5 procedure II.

This have to be repeated for the various levels of Time slots $(T)$ and the new connection request is allowed if it closes threshold value $\gamma_{N C}^{T H}$ during the period of time $(t)$ else it will be blocked.

\section{Performance Analysis}

The performance of the proposed scheduled CAC from the existing one can be highly differentiable by applying the values based on the queuing delay and by calculating the throughput from the service classes as for the different time slots $(t)$.

Here, there is a consideration of uplink transmission from Subscriber Station to a Base Station in a single-hop network. The performance of the proposed scheduled model coupling with the queuing algorithm is implemented using Network Simulator (NS2) (NS, 2014). This performance analysis is highly applied with the queuing algorithms that were supported under the implementation part of NS2, which was discussed under the section process II.

The following Fig. $3 a$ and $b$ shows the perspective of NS2 simulation model and NS2 configuration model respectively. Figure $3 \mathrm{a}$ demonstrates the simulation model with the 7 subscriber nodes requesting services from the single Base Station and Fig. $3 b$ demonstrates the configuration model with same set up in simulation model, where the corresponding node (6) requesting the service from the Base Station node $(0)$.

With the queuing delay, there is a value calculated from the different data rate of packets per second and average rate of packets to be serviced while arriving. By applying the scheduled CAC model along with the queuing algorithm, as the time slots $(t)$ increases with the data rate of packets, the queuing delay of packets should be decreases to achieve the quality in each and every class.

By calculating the average amount of data packets received at the destination node per given time slots without considering the retransmission of data packets even if loss occurs, the average throughput values for each and every service classes can be utilized. 


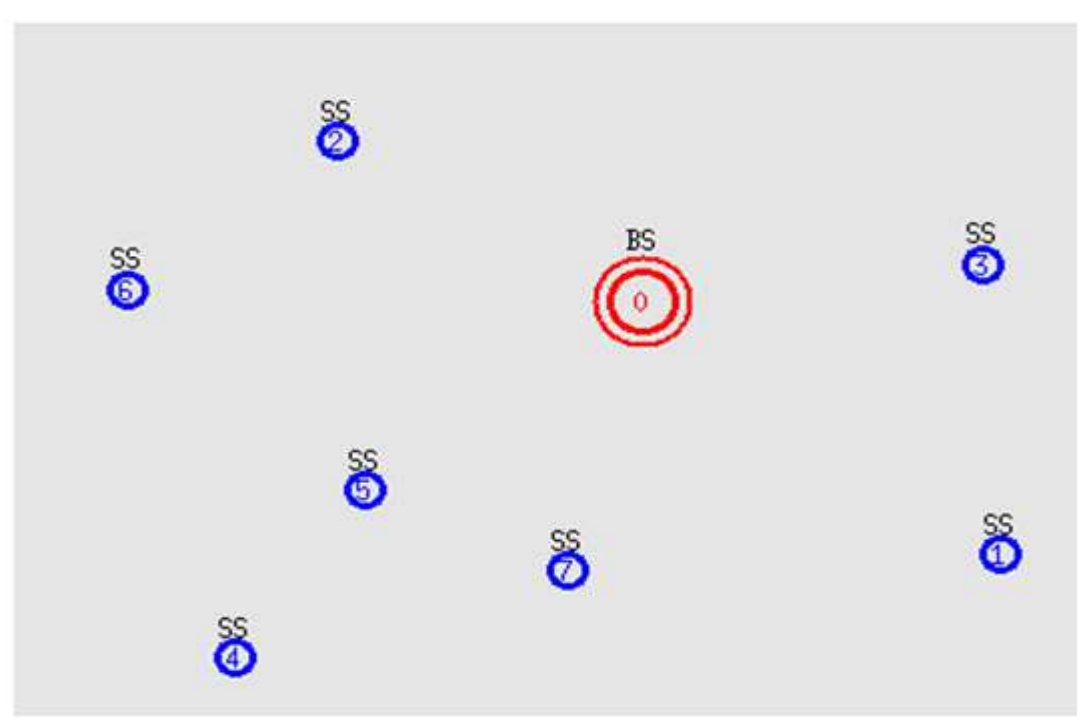

(a)

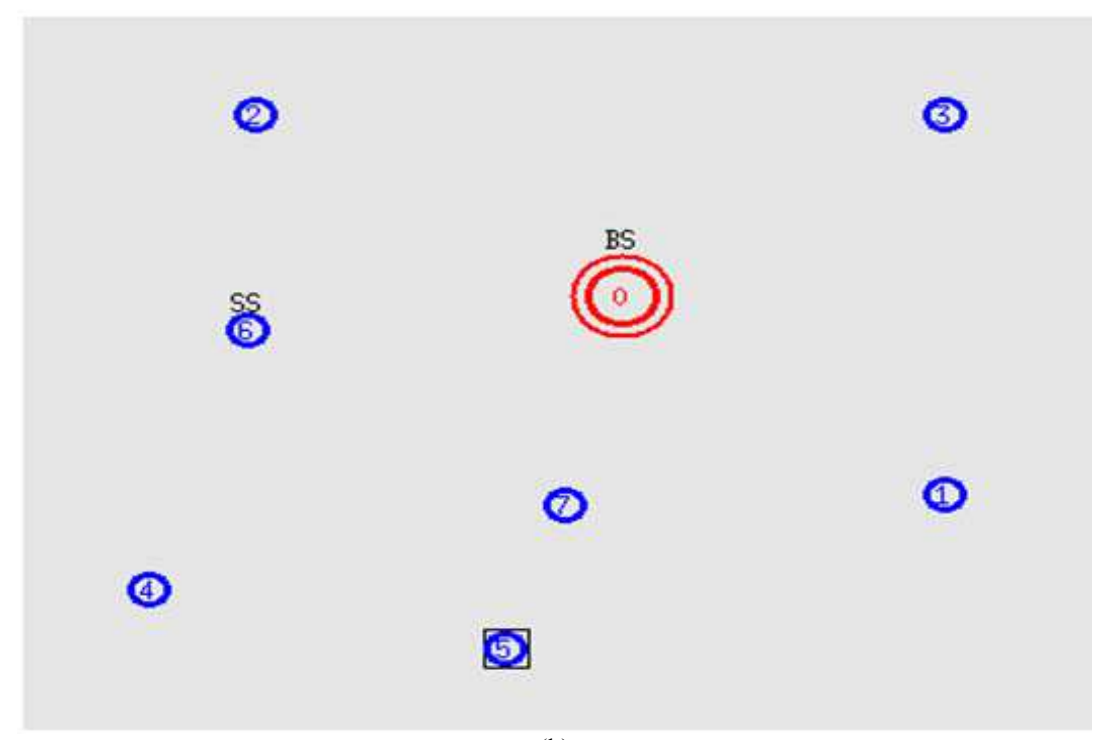

(b)

Fig. 3. (a) Simulation model (b) Configuration model

As the time slots ( $t$ ) increases, with the received data packet rate per time slot, the achievement of throughput could be increased to the level of Quality of Service.

We evaluate the performance of the scheduling algorithms under weighted service queuing approach and proposed scheduled CAC schemes in the following evaluation sections.

\section{WSQ Performance Evaluation}

The implementation has been simulated in NS2 (NS, 2014) for more than 1200 packets per slot to be considered with effective data rate of packets and their effect on weighted service queuing approach supports the scheduling algorithm for different service classes are evaluated. The estimated performance of proposed scheduling algorithm like DRR, FQ and SFQ supported by the NS2 has been compared with the existing weighted service queuing approach is plotted on the bar graph inserted on Fig. 4 by plotting the values between different scheduling algorithm to the scheduled packets per time slots with respective to different Service classes. We could find some mild variations in the performance of scheduling approaches which could bring effectiveness in achieving the Quality of Service in next generation wireless network systems. 


\section{Scheduled CAC Performance Evaluation}

Here, in this section we discuss about the performance evaluation of the proposed scheduled CAC with the usage of different service classes to achieve the Quality of Service. From Fathi et al. (2013), we have taken the predetermined departure rate values for $\gamma_{n r t P S}^{T H}$ as $900, \gamma_{B E}^{T H}$ as 500 whereas in class of $\gamma_{r t P S}^{T H}$ the value is evaluated less than 5 time slots we considered.

In terms of token bucket mechanism invoked on the proposed CAC Scheme, the data rate of packets is considered with the queuing approach by plotting the values between different service classes corresponding to the throughput and delay performance of the different queuing scheduling approaches.

By taking the time slots per packets into account, the variations can be predicted by considering the above parameters.
By considering the time slots up to 60 seconds, taken as $\mathrm{x}$ axis and the data rate of packets per slots up to 1200 packets taken as $\mathrm{y}$ axis the average throughput of the service class $\gamma_{r t P S}(t)$ is plotted and shown in Fig. 5a. On the other hand the queuing delay of the service class is plotted and shown in Fig. 5b.

Figure 6a and $6 \mathrm{~b}$ illustrates the effect of $\gamma_{n r t P S}(t)$ service class on the data rate of packets considering 1200 packets per slots with the parameters of average throughput and delay values respectively.

Figure $7 \mathrm{a}$ shows the plotted graph between the times per slots under $x$ axis whereas the data rate of packets under $\mathrm{y}$ axis to focus the performance of service class $\gamma_{B E}(t)$ on the average throughput increases to 1200 packets per slots.

Where, Fig. $7 \mathrm{~b}$ focuses on the effect of service class $\gamma_{\mathrm{BE}}$ on queuing delay by showing gradual decreases in the plotted graph values to achieve the Quality of Service in the next generation heterogeneous

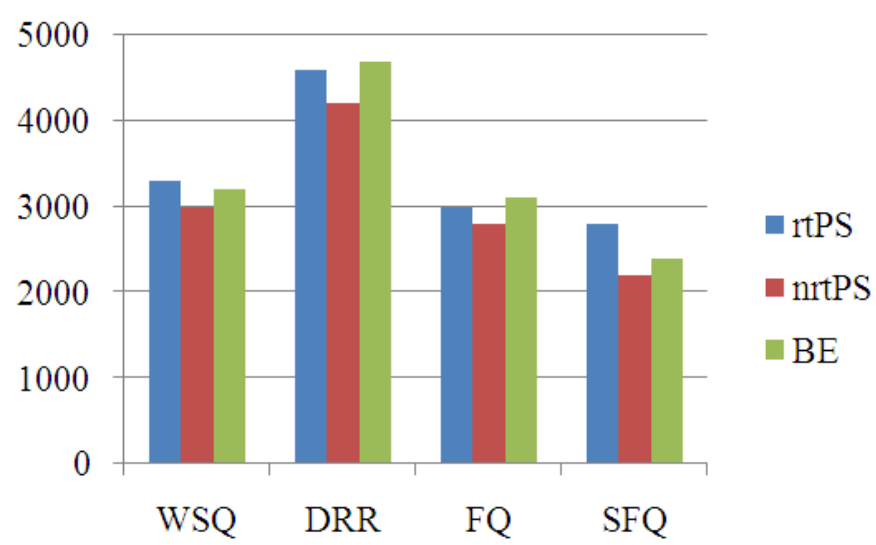

Fig. 4. Scheduled packets per time slots Vs. Scheduling algorithm

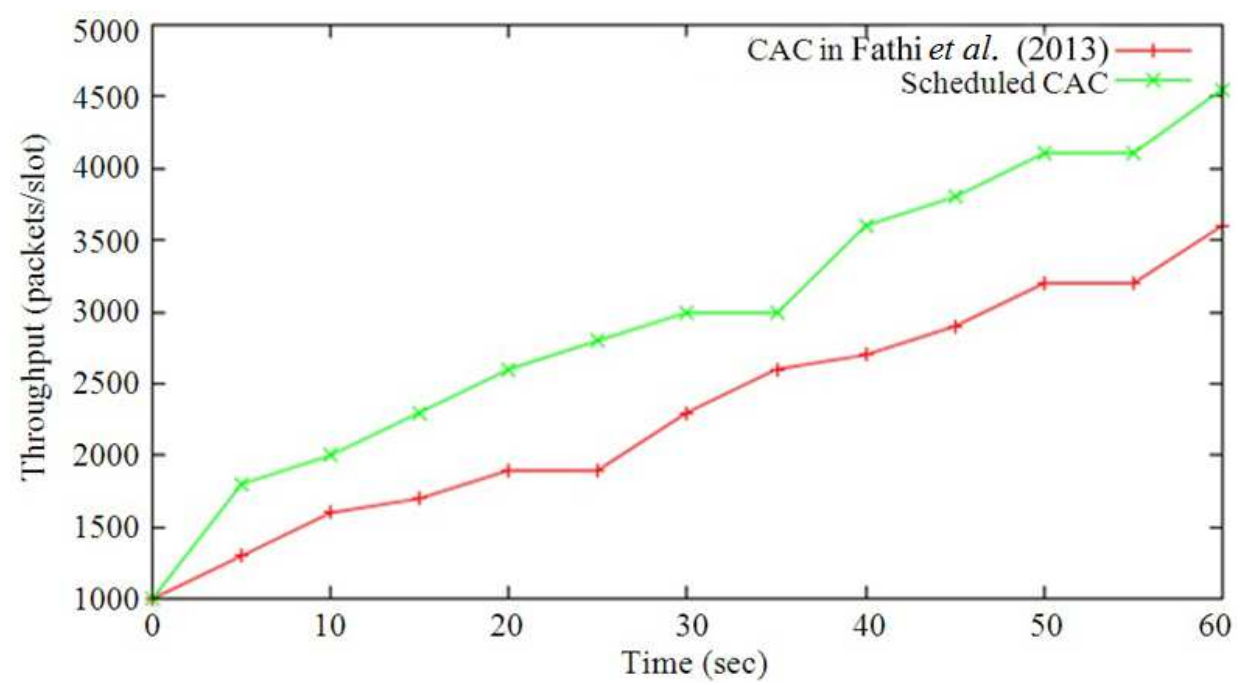

(a) 


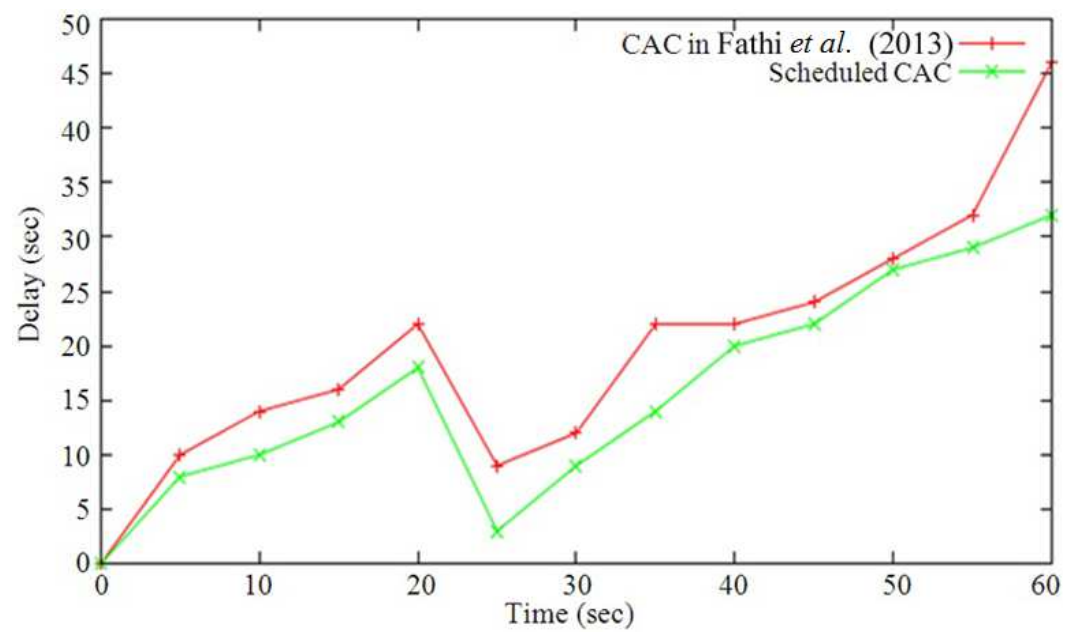

(b)

Fig. 5. (a) Time Vs. Throughput for rtPS service class (b) Time Vs. Delay for rtPS service class

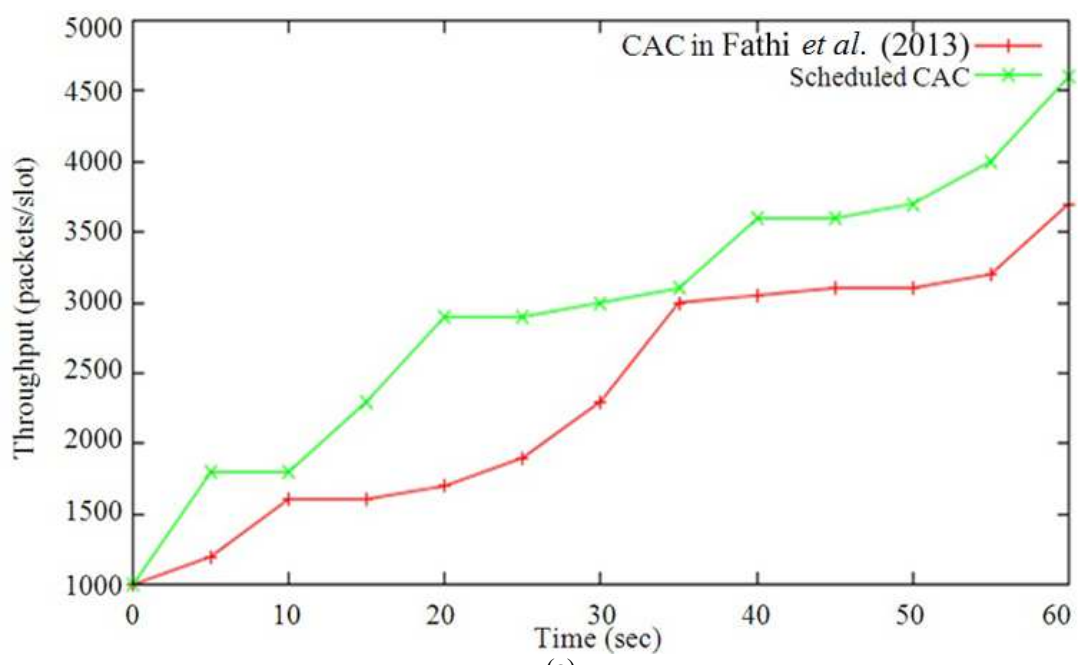

(a)

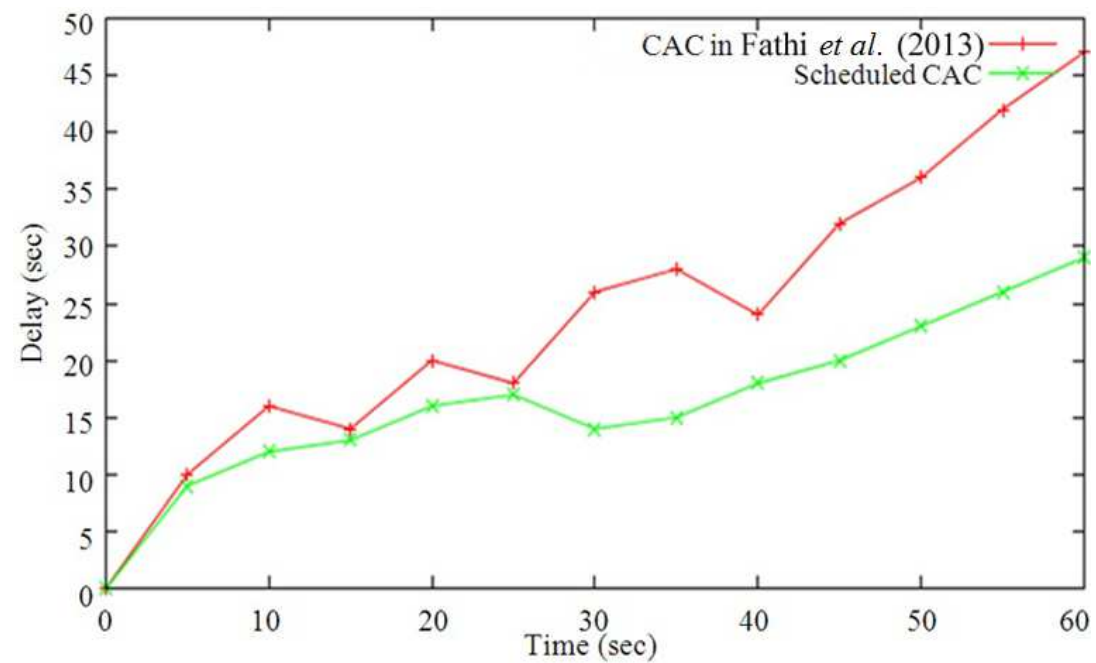

(b)

Fig. 6. (a) Time Vs. Throughput for nrtPS service class (b) Time Vs. Delay for nrtPS service class 


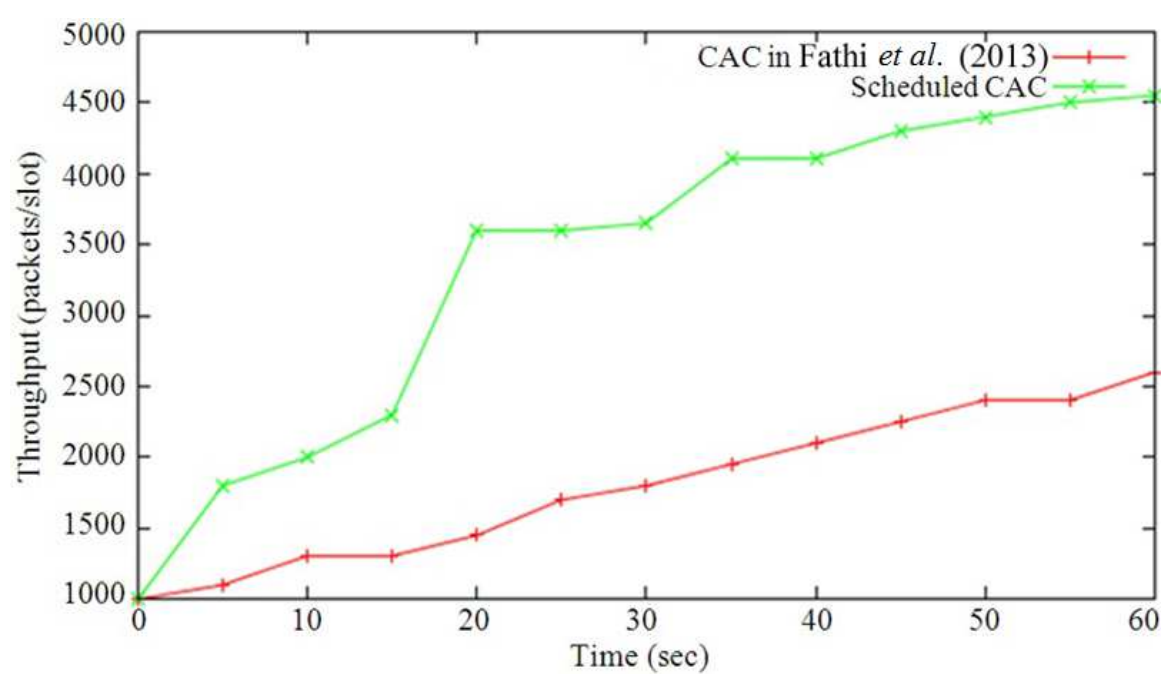

(a)

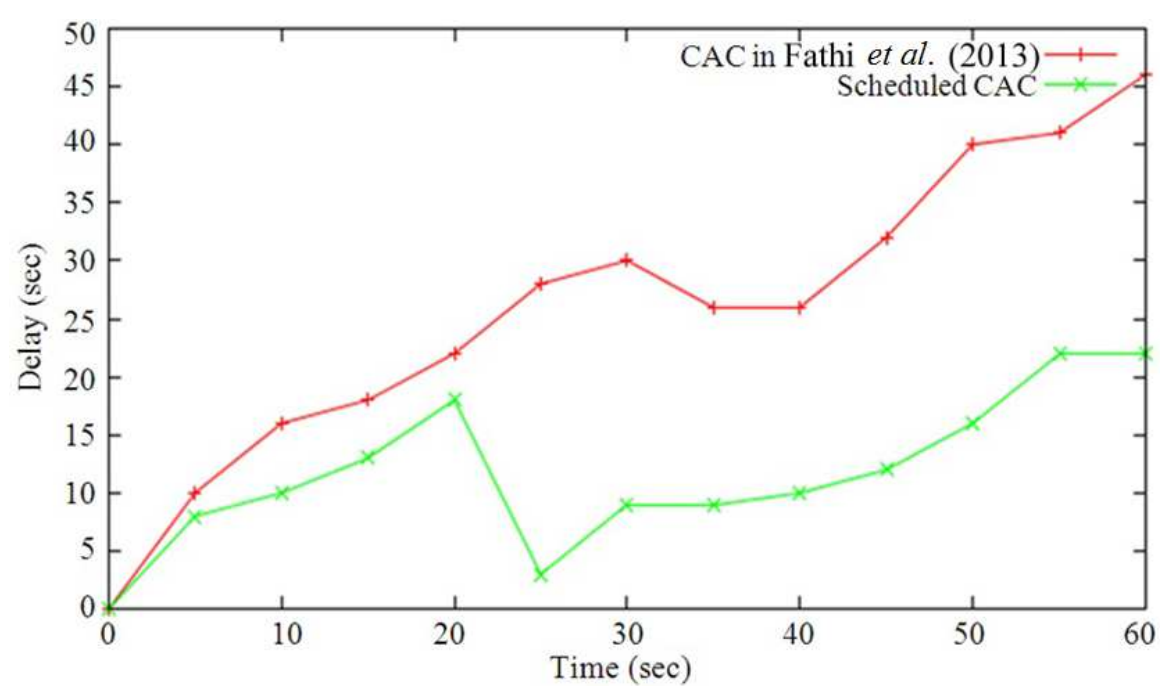

(b)

Fig. 7. (a) Time Vs. Throughput for BE service class. (b) Time Vs. Delay for BE service class

\section{Conclusion}

Over the next generation wireless network systems, the scheduling algorithm and proposed scheduled CAC model scheme with the additional usage of Token Bucket mechanism has been implemented and the performance metrics of throughput and queuing delay has been measured. Considering the weighted service queuing approach, the four types of service classes is worked on the parameters were throughput and delay and their effects was recorded the achievable Quality of Service with respective to the data packets per given time slots. The concept of allowing the new connection request could moreover achieve the better Quality of Service for the IEEE standards. There only limited numbers of service classes were considered to measure the performance of multimedia services. The scheduled CAC model will works only on these service classes. This is the major Limitation to be considered. The parameters were considered for the limited number of data packets per given time slots which could show variance in the performance of multimedia services if the number of data packets increases. As the requirements of services become restricted, the admitted connection of new request becomes more restricted as well. The future work of this research will concentrate on the CAC model, could achieve Quality of Service including different parameters such as packet loss, energy consumption, packet delivery ratio in heterogeneous wireless networks. 


\section{Acknowledgement}

I am so glad to convey my thanks to my research guide Prof. Dr. A. Arokiasamy, for his entire support throughout the whole process of paper to bring this study valuable to me by offering his worthful time, supervision and precious suggestions. I accomplished my work successfully only because of his great moral support and good direction. Last but not least, I would like to thank all my colleagues and friends for giving me wonderful support and encouragement during the preparation of this study.

\section{Author's Contributions}

All authors equally contributed in this work.

\section{Ethics}

This article is original and contains unpublished material. The corresponding author confirms that all of the other authors have read and approved the manuscript and no ethical issues involved.

\section{Refernces}

Fathi, M., S. Rashidi-Bajgan, A. Khalilzadeh and H. Taheri, 2013. A dynamic joint scheduling and call admission control scheme for IEEE 802.16 networks. Tele Commun. Syst., 52: 195-202. DOI: 10.1007/s11235-011-9555-8

Kolate, V.S., G.I. Patil and A.S. Bhide, 2012. Call admission control schemes and handoff prioritization in $3 \mathrm{G}$ wireless mobile networks. Int. J Eng. Innovative Technol., 1: 92-97.

Kumar, G.N. and A. Arokiasamy, 2014. Performance improvement of robust call admission control algorithm for QoS support over future generation wireless heterogeneous networks. J. Theoretical Applied Inform. Technol., 62: 605-605.
Marler, R.T. and J.S. Arora, 2004. Survey of multiobjective optimization methods for engineering. Structural Multidisciplinary Optimizat., 26: 369-395.

NS, 2014. Network Simulator.

Pla, V., J. Virtamo and J. Martínez-Bauset, 2008. Optimal robust policies for bandwidth allocation and admission control in wireless networks. Comput. Netw., 52: 3258-3272. DOI: 10.1016/j.comnet.2008.08.019

Ramesh Babu, H.S., G. Gowrishankar and P.S. Satyanarayana, 2010. Call admission control mechanism for optimal QoS in next generation wireless networks. Proceedings of the International Conference on Intelligent Systems, Modelling and Simulation, Jan. 27-29, IEEE Xplore Press, Liverpool, pp: 350-355. DOI: 10.1109/ISMS.2010.88

Rashidi-Bajgan, S., H. Taheri, M. Sabaghi and M. Fathi, 2010. An efficient call admission control for IEEE 802.16 networks. Proceedings of the 15th IEEE Workshop on Local and Metropolitan Area Networks, Jun. 10-13, IEEE Xplore Press, Princeton, NJ., pp: 361-365. DOI: 10.1109/LANMAN.2007.4295997

Deshmukh, S.B. and V.V. Deshmukh, 2013. Call admission control in cellular networks. Int. J. Adv. Res. Electrical, Electron. Instrument. Eng., 2: 1388-1391.

Tragos, E.Z., G. Tsiropoulos, G.T. Karetsos, S.A. KYriazakos, 2008. Admission control for QoS support in heterogeneous $4 \mathrm{G}$ wireless networks. IEEE Network, 22: 30-37. DOI: 10.1109/MNET.2008.4519962

Tsai, T.C. and C.H. Jiang, 2006. Token bucket based CAC and packet scheduling for IEEE 802.16 broadband wireless access networks. Proceedings of the 3rd IEEE Consumer Communications and Networking Conference, Jan. 8-10, IEEE Xplore Press, pp: 183-187. DOI: $10.1109 /$ CCNC.2006.1593012 